\title{
The Harmonic Bloch and Besov Spaces on the Real Unit Ball by an Oscillation
}

\author{
Xi Fu, ${ }^{1}$ Zhiyao Xu, ${ }^{2}$ and Xiaoyou Liu ${ }^{3}$ \\ ${ }^{1}$ Department of Mathematics, Shaoxing University, Shaoxing, Zhejiang 312000, China \\ ${ }^{2}$ Department of Mathematics, Jiangxi Vocational College of Industry and Engineering, Pingxiang, Jiangxi 337055, China \\ ${ }^{3}$ School of Mathematics and Physics, University of South China, Hengyang, Hunan 421001, China
}

Correspondence should be addressed to Xiaoyou Liu; liuxiaoyou2002@hotmail.com

Received 25 November 2015; Accepted 28 February 2016

Academic Editor: Ruhan Zhao

Copyright (c) $2016 \mathrm{Xi} \mathrm{Fu}$ et al. This is an open access article distributed under the Creative Commons Attribution License, which permits unrestricted use, distribution, and reproduction in any medium, provided the original work is properly cited.

Let $\mathbb{B}$ be the real unit ball in $\mathbb{R}^{n}$ and $f \in \mathscr{C}^{N}(\mathbb{B})$. Given a multi-index $m=\left(m_{1}, \ldots, m_{n}\right)$ of nonnegative integers with $|m|=N$, we set the quantity $\sup _{x \in \mathbb{B}, y \in E(x, r), x \neq y}\left(1-|x|^{2}\right)^{\alpha}\left(1-|y|^{2}\right)^{\beta}\left(\left|\partial^{m} f(x)-\partial^{m} f(y)\right| /|x-y|^{\gamma}[x, y]^{1-\gamma}\right), \quad x \neq y$, where $0 \leq \gamma \leq 1$ and $\alpha+\beta=N+1$. In terms of it, we characterize harmonic Bloch and Besov spaces on the real unit ball. This generalizes the main results of Yoneda, 2002 , into real harmonic setting.

\section{Introduction}

Let $\mathbb{B}$ be the real unit ball in $\mathbb{R}^{n}$ with $n \geq 2$, where $d v$ is the normalized volume measure on $\mathbb{B}$ and $d \sigma$ is the normalized surface measure on the unit sphere $S=\partial \mathbb{B}$. We denote the class of all harmonic functions on the unit ball by $H(\mathbb{B})$. For $f \in H(\mathbb{B}), \nabla f(x)$ denotes the gradient of $f$. Given a multiindex $m=\left(m_{1}, \ldots, m_{n}\right)$ of nonnegative integers, we use the notations $|m|=m_{1}+\cdots+m_{n}$ and

$$
\partial^{m} f=\frac{\partial^{|m|} f}{\partial x^{m}}=\frac{\partial^{|m|} f}{\partial x_{1}^{m_{1}} \cdots \partial x_{n}^{m_{n}}}
$$

For each $\alpha>0$, the harmonic $\alpha$-Bloch space $\mathscr{B}^{\alpha}$ consists of all functions $f \in H(\mathbb{B})$ such that

$$
\|f\|_{\alpha}=\sup _{x \in \mathbb{B}}\left(1-|x|^{2}\right)^{\alpha}|\nabla f(x)|<\infty,
$$

and the little $\alpha$-Bloch space $\mathscr{B}_{0}^{\alpha}$ consists of the functions $f \in$ $\mathscr{B}^{\alpha}$ such that

$$
\lim _{|x| \rightarrow 1^{-}} \sup _{x \in \mathbb{B}}\left(1-|x|^{2}\right)^{\alpha}|\nabla f(x)|=0
$$

The harmonic Besov space $\mathscr{B}_{p}$ is the space of all functions in $H(\mathbb{B})$ for which

$$
\int_{\mathbb{B}}\left(1-|x|^{2}\right)^{p}|\nabla f(x)|^{p} d \tau(x)<\infty,
$$

where $p>n-1$ and $d \tau(x)=\left(1-|x|^{2}\right)^{-n} d v(x)$ is the invariant measure on $\mathbb{B}$.

Let $f$ be a continuous function in $\mathbb{B}$. If there exists a constant $C$ such that

$$
\begin{aligned}
\mathscr{L}_{f}(x, y) & =\left(1-|x|^{2}\right)^{1 / 2}\left(1-|y|^{2}\right)^{1 / 2}\left|\frac{f(x)-f(y)}{x-y}\right| \\
& \leq C,
\end{aligned}
$$

for any $x, y \in \mathbb{B}$, then we say that $f$ satisfies weighted Lipschitz condition. By means of it, Ren and Kähler [1] obtained the following.

Theorem A. Let $f \in H(\mathbb{B})$. Then $f \in \mathscr{B}^{1}$ if and only if it satisfies the weighted Lipschitz condition.

Theorem B. Let $f \in H(\mathbb{B})$ and $p \in(2(n-1), \infty)$. Then $f \in$ $\mathscr{B}_{p}$ if and only if

$$
\iint_{\mathbb{B}} \mathscr{L}_{f}^{p}(x, y) d \tau(x) d \tau(y)<\infty .
$$


Let $\mathbb{D}$ be the open unit disk in the complex plane $\mathbb{C}$ and let $f$ be a continuous complex-value function in $\mathbb{D}$. Denote by

$$
M=\sup _{z \in \mathbb{D}, w \in D(z, r), z \neq w} \frac{\left(1-|z|^{2}\right)^{\alpha}\left(1-|w|^{2}\right)^{\beta}\left|\widehat{D}^{(n-1)} f(z)-\widehat{D}^{(n-1)} f(w)\right|}{|z-w|}
$$

where $D(z, r)$ is the hyperbolic disc with center $z \in \mathbb{D}$ and radius $r, n \geq 1$ an integer, $\alpha+\beta=n$, and $\widehat{D}^{(n)}=\partial^{n} / \partial z^{n}+$ $\partial^{n} / \partial \bar{z}^{n}$.

In [2], Yoneda characterized harmonic Bloch and Besov spaces in $\mathbb{D}$ in terms of $M$ as follows.

Theorem C. Let $f$ be a complex-value harmonic function in $\mathbb{D}$. Fix an integer $n \geq 1$ and a pair of real numbers $\alpha, \beta$ such that $\alpha+\beta=n$. Then $f \in \mathscr{B}^{1}(\mathbb{D})$ if and only if $M$ is bounded.

Theorem D. Let $f$ be a complex-value harmonic function in $\mathbb{D}$. Fix an integer $n \geq 2$ and a pair of real numbers $\alpha, \beta$ such that $\alpha+\beta=n$. Then for each $p \geq 1, f \in \mathscr{B}_{p}(\mathbb{D})$ if and only if

$$
\int_{\mathbb{D}} M^{p} d \lambda(z)<\infty
$$

where $d \lambda(z)=\left(1-|z|^{2}\right)^{-2} d z$.

See [3-5] for various characterizations of the Bloch, little Bloch, and Besov spaces in the unit ball of $\mathbb{C}^{n}$.

The main purpose of this paper is to give some characterizations for the spaces $\mathscr{B}^{\alpha}, \mathscr{B}_{0}^{\alpha}$, and $\mathscr{B}_{p}$ in the real unit ball along Yoneda's direction. In Section 2, we collect some known results that will be needed in the proof of our results. Our main results and their proofs are presented in Sections 3 and 4.

Throughout this paper, constants are denoted by $C$; they are positive and may differ from one occurrence to the other. The notation $A \asymp B$ means there is a positive constant $C$ such that $B / C \leq A \leq C B$.

\section{Preliminaries}

We will be using the same notation in $[1,6]$ : we write $x, y \in$ $\mathbb{R}^{n}$ in polar coordinates by $x=|x| x^{\prime}$ and $y=|y| y^{\prime}$. For any $a, b \in \mathbb{R}^{n}$, let

$$
[a, b]=|| a\left|b-a^{\prime}\right|
$$

Then the symmetry lemma in [7] shows that

$$
[a, b]=[b, a] \text {. }
$$

For any $a \in \mathbb{B}$, denote by $\phi_{a}$ the Möbius transformation in $\mathbb{B}$. It is an involution of $\mathbb{B}$ such that $\phi_{a}(0)=a$ and $\phi_{a}(a)=0$, which is of the form

$$
\phi_{a}(x)=\frac{|x-a|^{2} a-\left(1-|a|^{2}\right)(x-a)}{[x, a]^{2}}, \quad x \in \mathbb{B} .
$$

By simple computations, we have

$$
\begin{aligned}
\left|\phi_{a}(x)\right| & =\frac{|x-a|}{[x, a]}, \\
1-\left|\phi_{a}(x)\right|^{2} & =\frac{\left(1-|x|^{2}\right)\left(1-|a|^{2}\right)}{[x, a]^{2}} .
\end{aligned}
$$

For any $a \in \mathbb{B}$ and $r \in(0,1)$, we define the pseudohyperbolic ball with center $a$ and radius $r$ as

$$
E(a, r)=\left\{w \in \mathbb{B}:\left|\phi_{a}(w)\right|<r\right\} .
$$

Clearly, $E(a, r)=\phi_{a}(\mathbb{B}(0, r))$.

Lemma 1 (see [1, Lemma 2.1]). Let $r \in(0,1)$ and $y \in E(x, r)$. Then

$$
1-|x|^{2}=1-|y|^{2} \approx[x, y] \asymp|E(x, r)|,
$$

where $|E(x, r)|$ denotes the volume of $E(x, r)$.

The following is a characterization of the space $\mathscr{B}^{1}$ (resp., $\mathscr{B}_{0}^{1}$ ) which is proved in [8].

Lemma 2. Let $h \in H(\mathbb{B})$ and $N$ be a positive integer. Then $h \in \mathscr{B}^{1}$ (resp., $\mathscr{B}_{0}^{1}$ ) if and only if

$$
\begin{gathered}
\sup _{x \in \mathbb{B}}\left(1-|x|^{2}\right)^{N}\left|\partial^{m} h(x)\right|<\infty \\
\left(\text { resp. } \lim _{|x| \rightarrow 1^{-}} \sup _{x \in \mathbb{B}}\left(1-|x|^{2}\right)^{N}\left|\partial^{m} h(x)\right|=0\right)
\end{gathered}
$$

for all multi-index $m$ with $|m|=N$.

As an application of Lemma 2, we can obtain the following.

Lemma 3. Let $h \in H(\mathbb{B})$. Then $h \in \mathscr{B}^{1}$ if and only if for each $j \in\{1, \ldots, n\}$,

$$
\begin{aligned}
& \sup _{x, y \in \mathbb{B}, x \neq y} \frac{\left(1-|x|^{2}\right)\left(1-|y|^{2}\right)}{|x-y|}\left|\frac{\partial h}{\partial x_{j}}(x)-\frac{\partial h}{\partial x_{j}}(y)\right| \\
& <\infty .
\end{aligned}
$$

Proof. Fixing a point $x$ and letting

$$
y=x+t \nabla\left(\frac{\partial h}{\partial x_{j}}\right)(x) \longrightarrow x
$$


with $t \in \mathbb{R}$, we have

$$
\left(1-|x|^{2}\right)^{2}\left|\nabla\left(\frac{\partial h}{\partial x_{j}}\right)(x)\right| \leq L
$$

for each $j \in\{1, \ldots, n\}$. By Lemma 2 , we see that $h \in \mathscr{B}^{1}$.

For the converse, we assume that $h \in \mathscr{B}^{1}$. Let $h_{j}(x)=$ $\partial h(x) / \partial x_{j}$; then for each $j \in\{1, \ldots, n\}$

$$
\begin{aligned}
& \left|h_{j}(x)-h_{j}(y)\right|=\left|\int_{0}^{1} \frac{d h_{j}}{d s}(s x+(1-s) y) d s\right| \\
& \leq \sum_{k=1}^{n}\left|\left(x_{k}-y_{k}\right) \int_{0}^{1} \frac{\partial h_{j}}{\partial x_{k}}(s x+(1-s) y) d s\right| \\
& \quad \leq \sqrt{n}|x-y| \int_{0}^{1}\left|\nabla h_{j}(s x+(1-s) y)\right| d s \\
& \quad \leq C|x-y| \int_{0}^{1} \frac{d s}{\left(1-|s x+(1-s) y|^{2}\right)^{2}} .
\end{aligned}
$$

It follows from [9] that there exists $C>0$ such that

$$
\int_{0}^{1} \frac{d s}{\left(1-|s x+(1-s) y|^{2}\right)^{2}} \leq \frac{C}{\left(1-|x|^{2}\right)\left(1-|y|^{2}\right)} .
$$

This implies that

$$
\sup _{x, y \in \mathbb{B}, x \neq y} \frac{\left(1-|x|^{2}\right)\left(1-|y|^{2}\right)}{|x-y|}\left|h_{j}(x)-h_{j}(y)\right|<\infty .
$$

So the result follows.

Combining Theorem A and Lemma 3, we extend [2, Corollary 2.4] into the real harmonic setting as follows.

Corollary 4. Let $h \in H(\mathbb{B})$. Then for $i=1,2, h \in \mathscr{B}^{1}$ if and only if

$$
\begin{aligned}
& \sup _{x, y \in \mathbb{B}, x \neq y} \frac{\left(1-|x|^{2}\right)^{i / 2}\left(1-|y|^{2}\right)^{i / 2}}{|x-y|} \mid \partial^{i-1} f(x) \\
& -\partial^{i-1} f(y) \mid<\infty,
\end{aligned}
$$

where $\partial^{1} f=\partial f / \partial x_{j}$ for all $j \in\{1, \ldots, n\}$.

In the following, we give an example which shows that Corollary 4 does not hold for $i \geq 3$.

Example 5. Let $f(x)=\ln \left(\left(1-x_{1}\right)^{2}+x_{2}^{2}\right)$; then $f \in \mathscr{B}^{1}$. By a simple computation we have

$$
\begin{aligned}
& \frac{\partial^{2} f}{\partial x_{1}^{2}}=\frac{2}{\left(1-x_{1}\right)^{2}+x_{2}^{2}}-\frac{4\left(1-x_{1}\right)^{2}}{\left(\left(1-x_{1}\right)^{2}+x_{2}^{2}\right)^{2}}, \\
& \sup _{x \in \mathbb{B}} \frac{\left(1-|x|^{2}\right)^{3 / 2}}{|x|}\left|\frac{\partial^{2}}{\partial x_{1}^{2}} f(x)-\frac{\partial^{2}}{\partial x_{1}^{2}} f(0)\right|=\infty .
\end{aligned}
$$

\section{Results and Discussions}

3.1. Harmonic Bloch Spaces. In this section, we give some characterizations of the spaces $\mathscr{B}^{\alpha}$ which can be viewed as the generalizations of Yoneda's results into the real unit ball $\mathbb{B}$ of $\mathbb{R}^{n}$.

For a continuous function $h$ in $\mathbb{B}$ and $0 \leq \gamma \leq 1$, we write

$$
L \Lambda(h)=\frac{|h(x)-h(y)|}{|x-y|^{\gamma}[x, y]^{1-\gamma}}, \quad x, y \in \mathbb{B}, x \neq y .
$$

By using the notation, we characterize $\mathscr{B}^{1}, \mathscr{B}_{0}^{1}$ as follows.

Theorem 6. Let $f \in H(\mathbb{B}), N \geq 0$ be an integer and $0<r<$ 1. Then $f \in \mathscr{B}^{1}$ if and only if

$$
\begin{aligned}
L_{f} & =\sup _{x \in \mathbb{B}, y \in E(x, r), x \neq y}\left(1-|x|^{2}\right)^{\alpha}\left(1-|y|^{2}\right)^{\beta} L \Lambda\left(\partial^{m} f\right) \\
& <\infty
\end{aligned}
$$

for all multi-index $m$ with $|m|=N$, where $\alpha+\beta=N+1$.

Proof. First we prove the sufficiency. Let $f \in H(\mathbb{B})$. Then for each multi-index $m, \partial^{m} f \in H(\mathbb{B})$. For $r \in(0,1)$, it follows from [1] that

$$
\left|\nabla\left(\partial^{m} f\right)(x)\right| \leq \frac{C}{\left(1-|x|^{2}\right)} \int_{E(x, r)}\left|\partial^{m} f(y)\right| d \tau(y)
$$

Fixing $x \in \mathbb{B}$ and replacing $\partial^{m} f$ by $\partial^{m} f-\partial^{m} f(x)$, we have

$$
\begin{aligned}
& \left(1-|x|^{2}\right)^{|m|+1}\left|\nabla\left(\partial^{m} f\right)(x)\right| \\
& \leq C\left(1-|x|^{2}\right)^{|m|} \int_{E(x, r)}\left|\partial^{m} f(y)-\partial^{m} f(x)\right| d \tau(y) .
\end{aligned}
$$

By Lemma 1, we can deduce that

$$
\begin{aligned}
& \left(1-|x|^{2}\right)^{|m|+1}\left|\nabla\left(\partial^{m} f\right)(x)\right| \leq C\left(1-|x|^{2}\right)^{|m|+1} \\
& \cdot \int_{E(x, r)} \frac{\left|\partial^{m} f(y)-\partial^{m} f(x)\right|}{[x, y]} d \tau(y) \\
& \leq C \int_{E(x, r)}\left(1-|x|^{2}\right)^{\alpha}\left(1-|y|^{2}\right)^{\beta} \\
& \cdot L \Lambda\left(\partial^{m} f\right) d \tau(y) \leq C L_{f} \tau(\mathbb{B}(0, r)) .
\end{aligned}
$$

Since $\tau(\mathbb{B}(0, r))=n \int_{0}^{r} t^{n-1}\left(1-t^{2}\right)^{-n} d t$ is a constant, we see that

$$
\left(1-|x|^{2}\right)^{N+1}\left|\nabla\left(\partial^{m} f\right)(x)\right|<\infty,
$$

for any multi-index $m$ with $|m|=N$. Hence Lemma 2 yields that $f \in \mathscr{B}^{1}$. 
Now we prove the necessity. Let $y \in E(x, r), x \neq y$. Then for each multi-index $m$ with $|m|=N$, we have

$$
\begin{aligned}
& \left|\left(\partial^{m} f\right)(x)-\left(\partial^{m} f\right)(y)\right| \\
& \quad=\left|\int_{0}^{1} \frac{d\left(\partial^{m} f\right)}{d s}(s x+(1-s) y) d s\right| \\
& \quad \leq \sum_{k=1}^{n}\left|\left(x_{k}-y_{k}\right) \int_{0}^{1} \frac{\partial\left(\partial^{m} f\right)}{\partial x_{k}}(s x+(1-s) y) d s\right| \\
& \quad \leq \sqrt{n}|x-y| \int_{0}^{1}\left|\nabla\left(\partial^{(m)} f\right)(s x+(1-s) y)\right| d s \\
& \quad \leq C|x-y| \int_{0}^{1} \frac{d s}{(1-|x z+(1-s) y|)^{N+1}} .
\end{aligned}
$$

By Lemma 1 we infer that there exists $\lambda>0$ such that $1-|y|=$ $\lambda(1-|x|)$ and

$$
\begin{aligned}
L \Lambda\left(\partial^{m} f\right) & \leq C \int_{0}^{1} \frac{d s}{(s(1-|x|)+(1-s)(1-|y|))^{N+1}} \\
& \leq \frac{C}{\left(1-|x|^{2}\right)^{N+1}} \int_{0}^{1} \frac{d s}{[s+\lambda(1-s)]^{N+1}} \\
& \leq \frac{C}{\left(1-|x|^{2}\right)^{\alpha}\left(1-|y|^{2}\right)^{\beta}}
\end{aligned}
$$

Thus,

$$
\begin{aligned}
L_{f} & =\sup _{x \in \mathbb{B}, y \in E(x, r), x \neq y}\left(1-|x|^{2}\right)^{\alpha}\left(1-|y|^{2}\right)^{\beta} L \Lambda\left(\partial^{m} f\right) \\
& <\infty .
\end{aligned}
$$

So the proof is complete.

Theorem 7. Let $f \in H(\mathbb{B}), N \geq 0$ be an integer and $0<r<1$. Then $f \in \mathscr{B}_{0}^{1}$ if and only if

$$
\begin{aligned}
& \lim _{|x| \rightarrow 1^{-}} \sup _{x \in \mathbb{B}, y \in E(x, r), x \neq y}\left(1-|x|^{2}\right)^{\alpha}\left(1-|y|^{2}\right)^{\beta} L \Lambda\left(\partial^{m} f\right) \\
& \quad=0
\end{aligned}
$$

for all multi-index $m$ with $|m|=N$, where $\alpha+\beta=N+1$.

Proof. Sufficiency: assume that (33) holds. Then for any $\epsilon>0$, there exists $\delta \in(0,1)$ such that

$$
\sup _{x \in \mathbb{B}, y \in E(x, r), x \neq y}\left(1-|x|^{2}\right)^{\alpha}\left(1-|y|^{2}\right)^{\beta} L \Lambda\left(\partial^{m} f\right)<\epsilon
$$

whenever $\delta<|x|<1$. It follows from an argument similar to that in proof of Theorem 6 that we have

$$
\begin{aligned}
& \left(1-|x|^{2}\right)^{N+1}\left|\nabla\left(\partial^{m} f\right)(x)\right| \\
& \leq C \sup _{x \in \mathbb{B}, y \in E(x, r), x \neq y}\left(1-|x|^{2}\right)^{\alpha}\left(1-|y|^{2}\right)^{\beta} L \Lambda\left(\partial^{m} f\right) \\
& <C \epsilon,
\end{aligned}
$$

whenever $\delta<|x|<1$. Hence

$$
\begin{aligned}
& \lim _{|x| \rightarrow 1^{-}} \sup _{x \in \mathbb{B}, y \in E(x, r), x \neq y}\left(1-|x|^{2}\right)^{\alpha}\left(1-|y|^{2}\right)^{\beta} L \Lambda\left(\partial^{m} f\right) \\
& \quad=0
\end{aligned}
$$

from which we see that $f \in \mathscr{B}_{0}^{1}$.

Necessity: for $t \in(0,1)$, let $f_{t}(x)=f(t x)$. By Lemma 1 and proof of Theorem 6 , we see that, for each multi-index $m$ with $|m|=N$,

$$
\begin{aligned}
& \left(1-|x|^{2}\right)^{\alpha}\left(1-|y|^{2}\right)^{\beta} L \Lambda\left(\partial^{m}\left(f-f_{t}\right)\right) \\
& \leq C\left(1-|\xi|^{2}\right)^{N+1}\left|\nabla \partial^{m}\left(f-f_{t}\right)(\xi)\right|, \\
& \left(1-|x|^{2}\right)^{\alpha}\left(1-|y|^{2}\right)^{\beta} L \Lambda\left(\partial^{m} f_{t}\right) \\
& \leq \frac{C\left(1-|x|^{2}\right)^{\alpha+\beta}}{\left(1-|t|^{2}\right)^{N+1}}\left(1-|t \eta|^{2}\right)^{N+1}\left|\nabla\left(\partial^{m} f_{t}\right)(\eta)\right|
\end{aligned}
$$

for all $x \in \mathbb{B}$ and $\xi, \eta \in E(x, r)$. So

$$
\begin{aligned}
L_{f} \leq & C\left(1-|\xi|^{2}\right)^{N+1}\left|\nabla \partial^{m}\left(f-f_{t}\right)(\xi)\right| \\
+ & \frac{C\left(1-|x|^{2}\right)^{\alpha}\left(1-|y|^{2}\right)^{\beta}}{\left(1-|t|^{2}\right)^{N+1}}\left(1-|t \eta|^{2}\right)^{N+1} \\
& \cdot\left|\nabla\left(\partial^{m} f_{t}\right)(\eta)\right| .
\end{aligned}
$$

First letting $|x| \rightarrow 1^{-}$and then letting $t \rightarrow 1^{-}$, we obtain the desired result.

In the following, by removing the restriction $y \in E(x, r)$ in Theorem 6, we obtain the following.

Theorem 8. Let $f \in H(\mathbb{B}), 0 \leq \beta<1$, and $\beta \leq \alpha<1+\beta$. Then $f \in \mathscr{B}^{\alpha}$ if and only if

$$
Q=\sup _{x, y \in \mathbb{B}, x \neq y}\left(1-|x|^{2}\right)^{\beta}\left(1-|y|^{2}\right)^{\alpha-\beta} L \Lambda(f)<\infty .
$$

Proof. We only need to prove the necessity since the proof of sufficiency is similar to that in proof of Theorem 6. Assume that $f \in \mathscr{B}^{\alpha}$. For $x, y \in \mathbb{B}, s \in[0,1]$,

$$
\begin{aligned}
(1 & \left.-|s x+(1-s) y|^{2}\right)^{\alpha} \\
& \geq\left(s\left(\frac{1-|x|^{2}}{2}\right)+(1-s)\left(\frac{1-|y|^{2}}{2}\right)\right)^{\alpha} \\
& \geq\left(\frac{s}{2}\right)^{\beta}\left(\frac{1-s}{2}\right)^{\alpha-\beta}\left(1-|x|^{2}\right)^{\beta}\left(1-|y|^{2}\right)^{\alpha-\beta},
\end{aligned}
$$


which gives

$$
\begin{aligned}
& L \Lambda(f) \leq C \int_{0}^{1} \frac{d s}{\left(1-|s x+(1-s) y|^{2}\right)^{\alpha}} \\
& \leq C \int_{0}^{1} \frac{d s}{(s / 2)^{\beta}((1-s) / 2)^{\alpha-\beta}\left(1-|x|^{2}\right)^{\beta}\left(1-|y|^{2}\right)^{\alpha-\beta}} \\
& \leq \frac{C}{\left(1-|x|^{2}\right)^{\beta}\left(1-|y|^{2}\right)^{\alpha-\beta}} \int_{0}^{1} \frac{d s}{s^{\beta}(1-s)^{\alpha-\beta}} \\
& \leq \frac{C}{\left(1-|x|^{2}\right)^{\beta}\left(1-|y|^{2}\right)^{\alpha-\beta}},
\end{aligned}
$$

where the last integral converges since $\alpha<1+\beta$. Thus

$$
\left(1-|x|^{2}\right)^{\beta}\left(1-|y|^{2}\right)^{\alpha-\beta} L \Lambda(f)<\infty .
$$

Similarly, we can prove the following.

Theorem 9. Let $f \in \mathscr{B}^{\alpha}, 0 \leq \beta<1$, and $\beta \leq \alpha<1+\beta$. Then $f \in \mathscr{B}_{0}^{\alpha}$ if and only if

$$
\begin{aligned}
& \lim _{|x| \rightarrow 1^{-}} \sup _{x, y \in \mathbb{B}, x \neq y} \omega\left(\left(1-|x|^{2}\right)^{\beta}\left(1-|y|^{2}\right)^{\alpha-\beta}\right) L \Lambda(f) \\
& \quad=0 .
\end{aligned}
$$

3.2. Harmonic Besov Space. In order to prove our next result, we need the following lemma.

Lemma 10. Let $f \in H(\mathbb{B})$. Then $f \in \mathscr{B}_{p}$ if and only if

$$
\sup _{x \in \mathbb{B}}\left(1-|x|^{2}\right)^{N+1}\left|\nabla\left(\partial^{m} f\right)(x)\right| \in L^{p}(\mathbb{B}, d \tau)
$$

for all multi-index $m$ with $|m|=N$.

Proof. This follows from [10, Theorem 3.7] by letting $\alpha=p-$ $n$.

Lemma 11. Let $h \in H(\mathbb{B})$ and $0<r<1$. Then there exist constants $C>0, r<r^{\prime}<1$ such that

$$
\sup _{y \in E(x, r), x \neq y}\left|\frac{h(x)-h(y)}{x-y}\right| \leq C \int_{E\left(x, r^{\prime}\right)}|\nabla h(u)| d \tau(u) .
$$

Proof. By Cauchy's estimates and Lemma 1, for each $x \in \mathbb{B}$, we have

$$
\begin{aligned}
& \sup _{y \in E(x, r), x \neq y}\left|\frac{h(x)-h(y)}{x-y}\right| \leq C \sup _{\zeta \in E(x, r)}|\nabla h(\zeta)| \\
& \leq \frac{C}{\left|E\left(x, r^{\prime}\right)\right|} \int_{E\left(x, r^{\prime}\right)}|\nabla h(\zeta)| d v(\zeta) \\
& \quad \leq C \int_{E\left(x, r^{\prime}\right)}|\nabla h(\zeta)| d \tau(\zeta)
\end{aligned}
$$

for some $r^{\prime}>r$.
Now, we come to state and prove the result for harmonic Besov spaces.

Theorem 12. Let $f \in H(\mathbb{B}), N \geq 0$ be an integer and $0<r<$ 1. Then $f \in \mathscr{B}_{p}$ if and only if

$$
\begin{gathered}
K_{p}=\int_{\mathbb{B}}\left(\sup _{x \in \mathbb{B}, y \in E(x, r), x \neq y}\left(1-|x|^{2}\right)^{\alpha}\left(1-|y|^{2}\right)^{\beta}\right. \\
\left.\cdot L \Lambda\left(\partial^{m} f\right)\right)^{p} d \tau(x)<\infty,
\end{gathered}
$$

for all multi-index $m$ with $|m|=N$, where $\alpha+\beta=N+1$.

Proof. Let $f \in H(\mathbb{B})$. Suppose that

$$
\begin{aligned}
K_{p} & =\int_{\mathbb{B}} \sup _{x \in \mathbb{B}, y \in E(x, r), x \neq y}\left(1-|x|^{2}\right)^{\alpha}\left(1-|y|^{2}\right)^{\beta} \\
\cdot & L \Lambda\left(\partial^{m} f\right) d \tau<\infty .
\end{aligned}
$$

Set

$$
L_{f}(x)=\lim _{y \rightarrow x} \sup \left(1-|x|^{2}\right)^{\alpha}\left(1-|y|^{2}\right)^{\beta} L \Lambda\left(\partial^{m} f\right) .
$$

It follows from proof of Theorem 6 that we have

$$
\left(1-|x|^{2}\right)^{N+1}\left|\nabla\left(\partial^{m} f\right)(x)\right| \leq C L_{f}(x) .
$$

Since $L_{f}(x) \leq L_{f}$, we see that

$$
\begin{aligned}
& \int_{\mathbb{B}}\left(1-|x|^{2}\right)^{p(N+1)}\left|\nabla\left(\partial^{m} f\right)(x)\right|^{p} d \tau(x) \\
& \quad \leq C \int_{\mathbb{B}} L_{f}^{p}(x) d \tau(x) \leq C \int_{\mathbb{B}} L_{f}^{p} d \tau(x)=C K_{p},
\end{aligned}
$$

for all multi-index $m$ with $|m|=N$. By Lemma 10, $f \in \mathscr{B}_{p}$.

To prove the necessity, we suppose that $f \in \mathscr{B}_{p}$. By Lemmas 1 and 11, for each multi-index $m$ and $r<r^{\prime}<1$,

$$
\begin{aligned}
L_{f} & \leq C \sup _{x \in \mathbb{B}, y \in E(x, r), x \neq y}\left(1-|x|^{2}\right)^{\alpha+\beta} L \Lambda\left(\partial^{m} f\right) \\
& \leq C \int_{E\left(x, r^{\prime}\right)}\left(1-|u|^{2}\right)^{N+1}\left|\nabla\left(\partial^{m} f\right)(u)\right| d \tau(u) .
\end{aligned}
$$

Since

$$
\int_{E\left(x, r^{\prime}\right)} d \tau<\infty
$$

by Hölder's inequality and Fubini's theorem, we can obtain

$$
\begin{aligned}
K_{p} \leq C \int_{\mathbb{B}}\left(\int_{E\left(x, r^{\prime}\right)}\left(1-|u|^{2}\right)^{N+1}\right. \\
\left.\cdot\left|\nabla\left(\partial^{m} f\right)(u)\right| d \tau(u)\right)^{p} d \tau(x) \\
\leq C \int_{\mathbb{B}}\left(\left(1-|u|^{2}\right)^{N+1} \mid \nabla\left(\partial^{m} f\right)\right. \\
\cdot(u) \mid)^{p} d \tau(u) .
\end{aligned}
$$


It follows from Lemma 10 that we see that $K_{p}$ is bounded. This completes the proof.

\section{Conclusions}

In this paper, we characterize harmonic Bloch and Besov spaces by using the quantity $\sup _{x \in \mathbb{B}, y \in E(x, r), x \neq y}\left(1-|x|^{2}\right)^{\alpha}(1-$ $\left.|y|^{2}\right)^{\beta}\left(\left|\partial^{m} f(x)-\partial^{m} f(y)\right| /|x-y|^{\gamma}[x, y]^{1-\gamma}\right), x \neq y$. Since $\mid x-$ $y \mid \leq[x, y]$, our results can be viewed as the generalizations of Yoneda's results (see [2]) into real harmonic setting. Furthermore, we obtain a characterization of the space $\mathscr{B}^{\alpha}$ in terms of $Q=\sup _{x, y \in \mathbb{B}, x \neq y}\left(1-|x|^{2}\right)^{\beta}\left(1-|y|^{2}\right)^{\alpha-\beta} L \Lambda(f)$, where $0 \leq \beta<1, \beta \leq \alpha<1+\beta$.

\section{Competing Interests}

The authors declare that there is no conflict of interests regarding the publication of this paper.

\section{Acknowledgments}

The work was partly supported by National Natural Science Foundation of China (Grant nos. 11501374, 11501284), Natural Science Foundation of Zhejiang Province (Grant no. LQ14A010006), and Natural Science Foundation of Hunan Province (Grant no. 2015JJ6095).

\section{References}

[1] G. Ren and U. Kähler, "Weighted Lipschitz continuity and harmonic Bloch and BESov spaces in the real unit ball," Proceedings of the Edinburgh Mathematical Society, vol. 48, no. 3, pp. 743755, 2005.

[2] R. Yoneda, "The harmonic Bloch and Besov spaces by an oscillation," Proceedings of the Edinburgh Mathematical Society, vol. 45, pp. 229-239, 2002.

[3] S. Chen, S. Ponnusamy, and A. Rasila, "On characterizations of Bloch-type, Hardy-type and Lipschitz-type spaces," Mathematische Zeitschrift, vol. 279, no. 1-2, pp. 163-183, 2015.

[4] S. Chen and X. Wang, "On harmonic Bloch spaces in the unit ball of $\mathbb{C}^{n}$," Bulletin of the Australian Mathematical Society, vol. 84, no. 1, pp. 67-78, 2011.

[5] K. Zhu, Spaces of Holomorphic Functions in the Unit Ball, Springer, New York, NY, USA, 2005.

[6] L. V. Ahlfors, Möbius Transformations in Several Variables, University of Minnesota, 1981.

[7] S. Axler, P. Bourdon, and W. Ramey, Harmonic Function Theory, Springer, New York, NY, USA, 1992.

[8] B. R. Choe, H. Koo, and H. Yi, "Derivatives of harmonic Bergman and Bloch functions on the ball," Journal of Mathematical Analysis and Applications, vol. 260, no. 1, pp. 100-123, 2001.

[9] R. Zhao, "A characterization of Bloch-type spaces on the unit ball of $\mathbb{C}^{n}$," Journal of Mathematical Analysis and Applications, vol. 330, no. 1, pp. 291-297, 2007.

[10] E. S. Choi and K. Na, "Characterizations of the harmonic Bergman space on the ball," Journal of Mathematical Analysis and Applications, vol. 353, no. 1, pp. 375-385, 2009. 


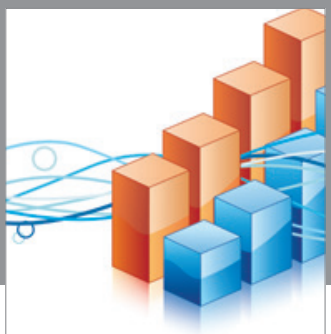

Advances in

Operations Research

vatem alat4

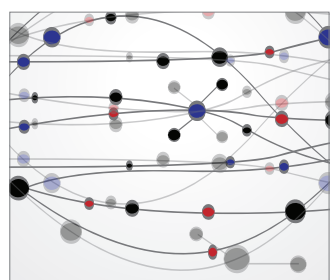

\section{The Scientific} World Journal
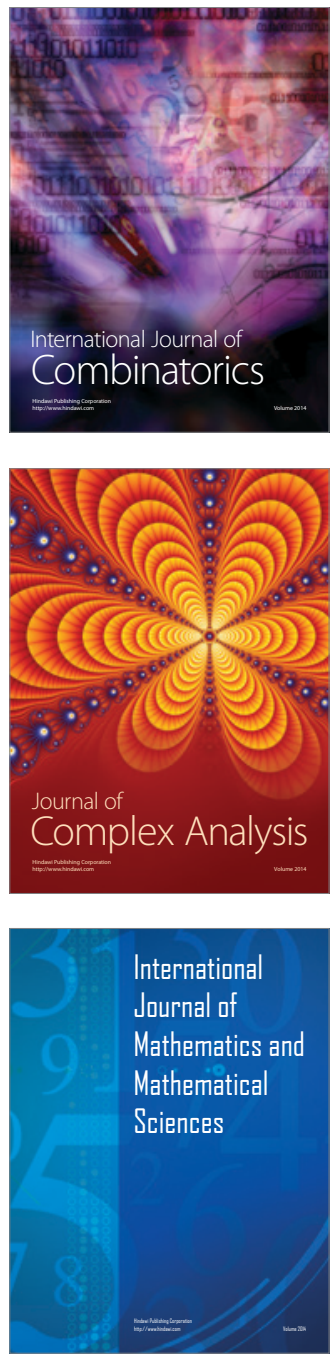
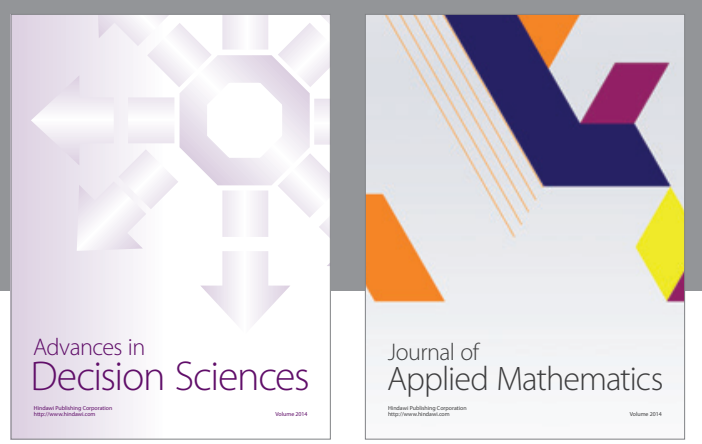

Algebra

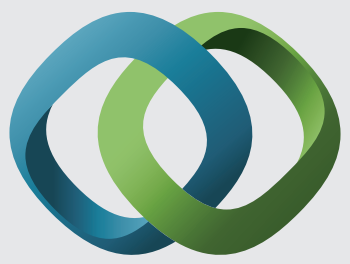

\section{Hindawi}

Submit your manuscripts at

http://www.hindawi.com
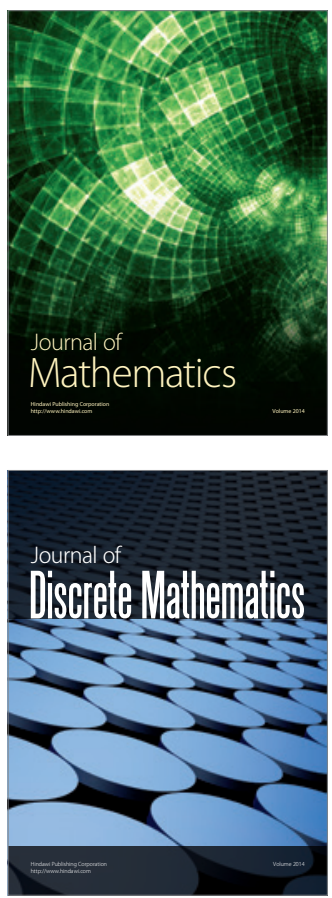

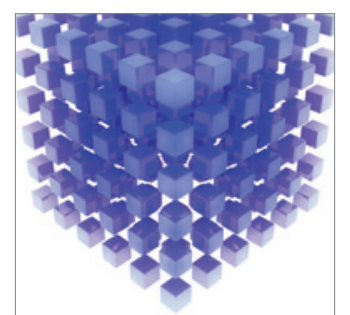

Mathematical Problems in Engineering
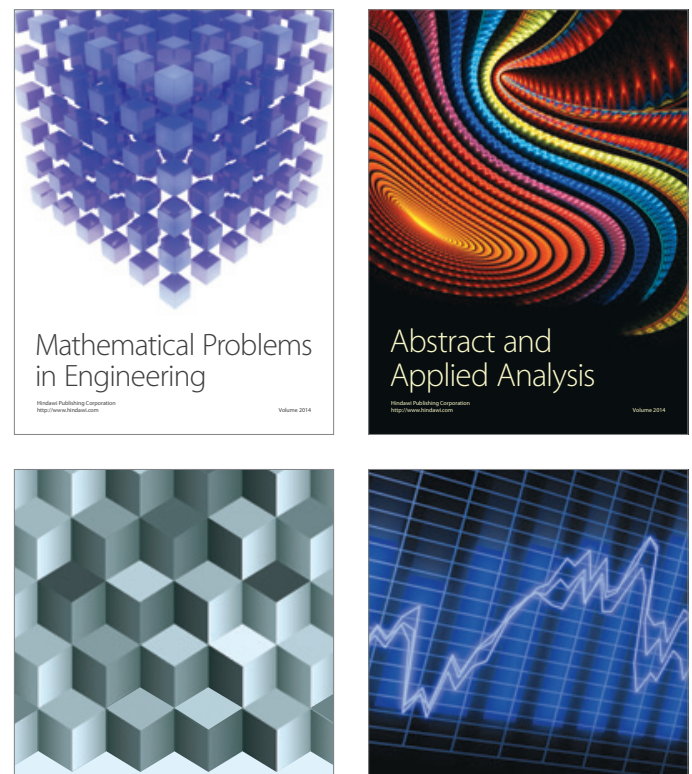

Journal of

Function Spaces

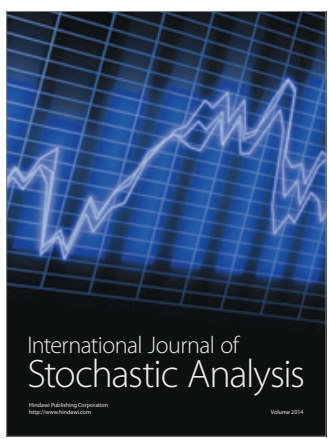

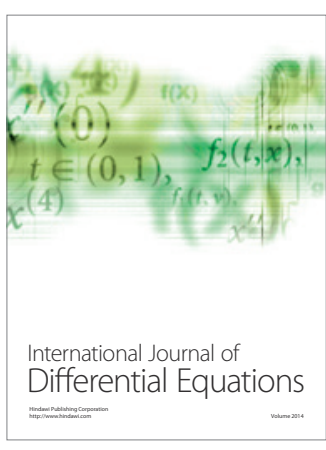
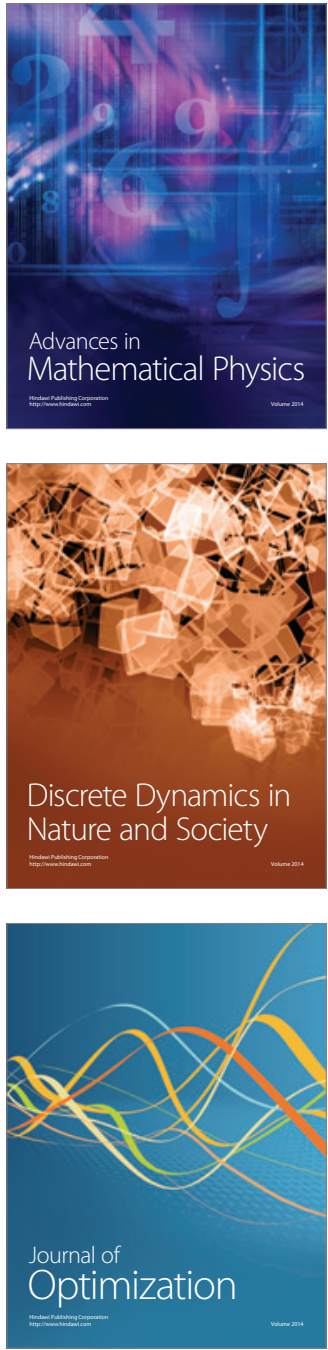